\title{
Measurement of the $B$ Cross Section at CDF via $B$ Semileptonic Decays
}

\author{
The CDF Collaboration
}

Fermi National Accelerator Laboratory

P.O. Box 500, Batavia, Illinois 60510

May 1994

Submitted to the 27th International Conference on High Energy Physics, Glasgow, Scotland, July 21-27, 1994 


\section{Disclaimer}

This report was prepared as an account of work sponsored by an agency of the United States Government. Neither the United States Government nor any agency thereof, nor any of their employees, makes any warranty, express or implied, or assumes any legal liability or responsibility for the accuracy, completeness, or usefulness of any information, apparatus, product, or process disclosed, or represents that its use would not infringe privately owned rights. Reference herein to any specific commercial product, process, or service by trade name, trademark, manufacturer, or otherwise, does not necessarily constitute or imply its endorsement, recommendation, or favoring by the United States Government or any agency thereof. The views and opinions of authors expressed herein do not necessarily state or reflect those of the United States Government or any agency thereof. 
ICHEP94 gls0092

Submitted to Pa-02 Pl-19

FERMILAB-CONF-94/134-E

ILL P-94-05-037

May 30, 1994

\title{
Measurement of the $B$ Cross Section at CDF via $B$ Semileptonic Decays
}

\author{
The CDF Collaboration ${ }^{\dagger}$
}

\begin{abstract}
Using data collected during the 1992-1993 collider run at Fermilab, CDF has reconstructed several hundred charmed mesons $\left(D^{0}, D^{+}, D^{*+}\right.$ and $\left.D_{s}\right)$ in association with leptons from $B$ semileptonic decays. We report on a measurement of the cross section of $B$ and $B_{s}$ mesons as a function of transverse momentum using this sample. The observation of a charmed meson eliminates many systematic uncertainties in the background subtraction inherent in previous measurements from inclusive lepton samples, and allows the backgrounds to be measured from the data. The $B$ meson $p_{T}$ range probed by the lepton+charm technique is $18 \mathrm{GeV}$ and above, and thus these measurements complement similar measurements at lower $p_{T}$ in the fully exclusive channels $B \rightarrow J / \psi K$ and $B \rightarrow J / \psi K^{*}$. Results are compared to other Tevatron measurements and Next-To-Leading-Order QCD predictions.
\end{abstract}

Submitted to the 27th International Conference on High Energy Physics, Glasgow, Scotland, 20-27 July, 1994

$\dagger$ Contact person: Thomas J. LeCompte (lecompte@fnald.fnal.gov), University of Illinois at Urbana-Champaign, Loomis Laboratory of Physics, $1110 \mathrm{~W}$. Green St.,Urbana, IL 61801 



\section{Introduction}

The Collider Detector at Fermilab (CDF) is designed to study high transverse momentum physics, with a particular emphasis on high $p_{T}$ leptons (for example, from $W \rightarrow l \nu)$. Besides $W$ 's, $Z$ 's and top, another source of leptons is semileptonic $B$ decays. Because semileptonic $B$ decays have much larger branching fractions than exclusive decays involving $J / \psi$ 's (especially after requiring the decay $J / \psi \rightarrow \mu^{+} \mu^{-}$) we can explore rarer processes: in particular, production of $B$ mesons at high $p_{T}$ $(\geq 18 \mathrm{GeV})$, where the cross section is a few nanobarns per $\mathrm{GeV}$.

Measuring the cross section from the inclusive lepton spectrum requires the calculation and subtraction of rather large backgrounds, leading to substantial uncertainties. Requiring an identified charmed particle in the final state not only greatly reduces the background, but also provides a mechanism to measure it: a $B$ meson will produce a $\bar{D}$ with a positive lepton, so $\bar{D}$ candidates with negative leptons provide a measure of the background. (In this paper, reference to any particular state implies the charge conjugate state as well.)

Muons and electrons each have advantages and disadvantages. For the CDF detector, the geometric acceptance for central electrons is substantially larger than for central muons, especially when the muon is required to pass through both sets of muon chambers. Furthermore, $\pi$ and $K$ decays provide an additional non- $B$ source of muons. On the other hand, electron identification requirements $(e . g . E / p)$ introduce implicit isolation cuts with efficiencies that depend on the decay and fragmentation of the $b$ quark, as well as characteristics of the underlying event. Electrons can provide a larger, and potentially purer $B$ sample, but muon identification is more efficient than electron identification.

This paper describes a measurement of $B$ and $B_{s}$ production using $17.9 \pm 0.6 \mathrm{pb}^{-1}$ of $\bar{p} p$ collisions at $\sqrt{s}=1.8 \mathrm{TeV}$ collected with the CDF detector between August, 1992 and May, 1993. The $B$ cross section is measured from the number of $D^{0}$ 's and $D^{*+}$ 's reconstructed in the inclusive muon sample. The $B_{s}$ fraction is measured from the ratio of $D_{s}$ to $D^{+}$events in a combined electron and muon sample.

\section{Detector}

The CDF detector is described in detail elsewhere [1]. The central muon system (including the upgrade chambers installed in 1992) and the silicon vertex detector (also installed in 1992) are briefly described below. 
The central muon chambers (CMU) consist of four layers of limited streamer chambers located behind the central calorimeters (approximately $5 \lambda$ thick). These chambers measure the azimuth $(\phi)$ of the muon from drift times and the polar angle $(\theta)$ of the muon using charge division. The CMU covers the pseudorapidity range $|\eta|<0.6$ and can be reached with muons with transverse momentum above $1.4 \mathrm{GeV}$. For this run, new central muon upgrade chambers (CMP) were added behind an additional $60 \mathrm{~cm}$ of steel. These chambers consist of four layers of drift chambers that measure the $\phi$ position. Approximately $53 \%$ of the solid angle for $|\eta|<0.6$ is covered by both CMU and CMP chambers. Both chambers reconstruct short tracks of up to four hits each called "stubs".

Surrounding the $1.9 \mathrm{~cm}$ radius beampipe is a four layer silicon microvertex detector (SVX) that is $51 \mathrm{~cm}$ long and consists of two identical cylindrical modules which meet at the detector midplane. Because $\bar{p} p$ interactions are spread along the beamline with standard deviation of about $30 \mathrm{~cm}$, just over half of the events originate from primary vertices inside the SVX fiducial region. Single hit resolution has been measured to be $\sigma=13 \mu \mathrm{m}$ and impact parameter resolution at high momentum has been measured to be $\sigma=17 \mu \mathrm{m}$.

\section{$3 \quad B$ Cross section with $\mu+D^{0}$ and $\mu+D^{*+}$}

\subsection{Muon Triggering and Identification}

CDF uses a three tiered triggering scheme. At Level One, we require a CMU stub with slope consistent with detection of a high $p_{T}$ muon. Additionally, confirmation (at least 2 of 4 hits) in the CMP chamber is required. At Level Two, 2-dimensional tracking information ( $\phi$ view only) is available and we require that there be a high $p_{T}$ track pointing towards the muon stub. Finally, the Level Three trigger is based on a processor farm running a special version of the offline reconstruction program; we require that a $7.5 \mathrm{GeV}$ track be found and matched to a CMU stub (again, with CMP confirmation) within $10 \mathrm{~cm}$ in the $\phi$ direction.

In this analysis, we identify muons as central tracking chamber (CTC) tracks that point to stubs in both the inner CMU and outer CMP muon chambers. Additionally, we impose a momentum dependent matching requirement on the track-stub combination: the $\chi^{2}$ of the match must be less than 9 for the $\phi$ view of both chambers and less than 12 for the $\theta$ view in the CMU chambers. From $Z \rightarrow \mu^{+} \mu^{-}$and $J / \psi \rightarrow \mu^{+} \mu^{-}$ data, we can measure the efficiency of the muon reconstruction and the requirements for matching between the tracking chamber and the muon chambers. The combined 
efficiency is $96.6 \pm 0.4 \%$. Additionally, we require the muon $p_{T}$ to be no less than 7.6 $\mathrm{GeV}$, to avoid the sharp turn-on of the Level Three trigger.

\subsection{Charm Reconstruction}

The analysis starts with an identified muon as a seed. We select for further analysis charged particle tracks in the event that pass minimal quality cuts. The invariant mass of $\mu$-track combination is calculated, and the track is selected for further analysis if the mass is less than the $B$ meson mass. (Taken to be $5.3 \mathrm{GeV}$ to allow for the detector resolution)

Once the candidate tracks have been selected, they are assigned kaon and pion masses, and $\left(K^{-} \pi^{+}\right)$and $\left(K \pi^{+} \pi^{+}\right)$invariant masses are computed. Should the $(K \pi)$ combination have a mass between 1.55 and $2.25 \mathrm{GeV}$, and make a mass with the $\mu$ of less than $5.3 \mathrm{GeV}$, it is considered a $D^{0}$ candidate, and the mass is recalculated applying the constraint that the two tracks intersect at a common point. In addition, should the difference in mass of the $\left(K^{-} \pi^{+} \pi^{+}\right)$and $\left(K^{-} \pi^{+}\right)$systems be less than 153 $\mathrm{MeV}$, the event is identified as containing a $D^{*+}$ candidate as well.

We do not make any SVX tracking requirement, nor do we make any lifetime requirement; we do not want to induce a correlation between cross-section and lifetime measurements.

\subsection{Acceptance and Kinematic Correction}

In $B$ semileptonic decays, there is always an undetected neutrino, and occasionally missing photons or pions as well, for example in the decay $B^{-} \rightarrow \mu^{-} \nu D^{* 0}$ followed by $D^{* 0} \rightarrow D^{0} \pi^{0}$ or $D^{0} \gamma$. Because we only partially reconstruct the $B$, we must estimate the $B$ meson $p_{T}$ from kinematic properties of detected particles. We have chosen two variables to parameterize this correction, the mass of the lepton $+D^{0}$ or $D^{*}, m_{v i s i b l e}$ and the transverse momentum of the lepton $+D^{0}$ or $D^{*}$ system, $p_{\text {Tvisible }}$.

This correction is applied in two steps. First, the visible $p_{T}$ is multiplied by the following function:

$$
1+a_{1} x+a_{2} x^{2}+a_{3} x^{3}+a_{4} x^{4}
$$

where $x$ is the missing mass fraction

$$
x=\frac{m_{B}-m_{\text {visible }}}{m_{B}}
$$


Next, the partially corrected $p_{T}$ is further corrected using the following prescription:

$$
p_{T}(\text { fully corrected })=\frac{b_{1}}{1-b_{2} p_{T}(\text { mass corrected })}
$$

The parameters of the correction functions are obtained by comparing the reconstructed $m_{v i s i b l e}$ and $p_{T \text { visible }}$ from a sample of two million Monte Carlo events with the generated $B$ meson $p_{T}$. This Monte Carlo sample was generated for $b$ quark $p_{T} \geq 15 \mathrm{GeV}$ using the DFLM parton distribution and Peterson fragmentation $(\epsilon=0.006)$. After all offline cuts are made, approximately $12,000 D^{0}$ 's and 4,000 $D^{*+}$ 's are reconstructed. The correction parameters are shown in the following table:

\begin{tabular}{||l|r|r||}
\hline Parameter & $B \rightarrow D^{0} \mu X$ analysis & $B \rightarrow D^{*+} \mu X$ analysis \\
\hline$a_{1}$ & 1.0969 & 0.9367 \\
\hline$a_{2}$ & -0.14128 & 1.2514 \\
\hline$a_{3}$ & -8.3275 & -11.3718 \\
\hline$a_{4}$ & 11.738 & 13.280 \\
\hline$b_{1}$ & 0.8994 & 0.88856 \\
\hline$b_{2}$ & 0.000330 & 0.000384 \\
\hline
\end{tabular}

Figure 1 shows the ratio of the generated $B$ meson $p_{T}$ to reconstructed $p_{T}$ after all corrections have been applied as a function of generated $B$ meson $p_{T}$. The distribution is flat in the region of interest (18-34 GeV), and has a value of about one, by construction. We can determine the $B$ meson momentum to about $15 \%$ on an eventby-event basis. Due to this $15 \%$ resolution, we have some sensitivity to the generated $B$ meson spectrum: a difference between the true and generated $B p_{T}$ spectra would manifest itself as an error in the cross section. If one replaces the generated spectrum by one that is a factor two smaller for every $4 \mathrm{GeV}$ increase in $p_{T}$, the cross section changes by less than $4 \%$ per bin.

By applying a correction based on the invariant mass of the $\mu+D$ system, we rcmove a large fraction of the uncertainties associated with the $D^{* *}$ composition of $B$ semileptonic decays. Varying the Monte Carlo $D^{* *}$ fraction from $20 \%$ to $10 \%$ changes the corrected $B$ meson $p_{T}$ by less than $0.5 \%$.

In addition to the kinematic corrections, we also use the same Monte Carlo sample to calculate the reconstruction efficiency and geometric acceptance. The combined acceptance and efficiency as a function of generated $B$ meson $p_{T}$ are shown in Figure 2. 


\section{4 $B$ Cross Section with $\mu+D^{0}$}

To obtain a $B \rightarrow \mu D^{0}$ sample, we make the following requirements on the $K \pi$ combination:

- $q(K)=q(\mu)$ (i.e. the charge is consistent with $B$ decay)

- $m(\mu K \pi) \leq 5.3 \mathrm{GeV}(m(B))$

- $p_{T}(K) \geq 1.5 \mathrm{GeV}$

- $p_{T}(\pi) \geq 1.5 \mathrm{GeV}$

- $p_{T}(K)$ or $p_{T}(\pi) \geq 3.0 \mathrm{GeV}$

- $\left|\cos \theta^{*}\right| \leq 0.8$

Here $\theta^{-}$is the angle between the kaon and the muon in the $(K \pi)$ rest frame. Because the $D$ is a pseudoscalar, the distribution for real $D$ decays is flat but the jet background (where the muon is from punchthrough or $\pi$ or $K$ decays) is forwardbackward peaked. Note that we do not reject identified $D^{*+}$ 's: this is a measurement of the $B$ cross section via the inclusive decay $B \rightarrow \mu D^{0} X$.

We fit the $K \pi$ mass spectrum to a Gaussian on a quadratic background. The mean of the Gaussian is fixed to the known $D^{0}$ mass.[3] The $K \pi$ mass spectrum from the sample with the wrong lepton charge is simultaneously fit: the two backgrounds are constrained to be the same and the width of the peak in the wrong sign distribution is determined from the Monte Carlo width of the $D^{0}$ reflection when the $K$ and $\pi$ assignments are reversed. We exclude the region below $1.72 \mathrm{GeV}$, to avoid the $D^{0}$ decay to $K^{-} \pi^{+} \pi^{0}$ ). In short, there are five parameters of the simultaneous fits of the right and wrong sign $K \pi$ spectrum: three for the quadratic background, the width of the $D^{0}$, and the number of $D^{0}$ 's. Figure 3 shows the $K \pi$ mass spectrum for the right (top) and wrong (bottom) sign lepton combination.

We then divide the sample into three bins of $B$ meson $p_{T}$ and repeat the process. Here the widths of the Gaussians are constrained to the value returned by the Monte Carlo, scaled by the ratio of the inclusive $D^{0}$ width to the Monte Carlo prediction, so there are only four fit parameters.

The numbers of reconstructed $D^{0}$ 's and the single species $B$ cross sections derived from these numbers are shown in the following table. We assume that $\sigma\left(B^{0}\right)=$ $\sigma\left(B^{+}\right)=\sigma\left(\overline{B^{0}}\right)=\sigma\left(B^{-}\right)$. 


\begin{tabular}{||l|r|r||}
\hline$p_{T}$ range & Events reconstructed & $d \sigma / d p_{T}|y|<1(\mathrm{nb} / \mathrm{GeV})$ \\
\hline All & $459 \pm 69$ & - \\
\hline $18-22 \mathrm{GeV}$ & $112 \pm 26$ & $12.1 \pm 2.8 \pm 2.0$ \\
\hline $22-26 \mathrm{GeV}$ & $98 \pm 22$ & $5.7 \pm 1.3 \pm 1.0$ \\
\hline $26-34 \mathrm{GeV}$ & $98 \pm 23$ & $2.0 \pm 0.5 \pm 0.3$ \\
\hline
\end{tabular}

where the sources of systematic uncertainty are listed in the following table:

\begin{tabular}{||l|r||}
\hline Source of Uncertainty & Uncertainty \\
\hline Luminosity & $3.6 \%$ \\
\hline Acceptance & $6 \%$ \\
\hline Tracking efficiency & $2 \%$ \\
\hline Trigger efficiency & $3 \%$ \\
\hline$K, \pi$ decays in flight & $2 \%$ \\
\hline$B p_{T}$ resolution & $4 \%$ \\
\hline Fitting method and background shape & $8 \%$ \\
\hline Branching ratio $(B \rightarrow \mu X)$ & $10 \%$ \\
\hline Branching ratio $\left(B \rightarrow \mu D^{* *}\right)$ & $2 \%$ \\
\hline Branching ratio $(D \rightarrow K \pi)$ & $6 \%$ \\
\hline Total & $17 \%$ \\
\hline
\end{tabular}

\section{5 $B$ Cross Section with $\mu+D^{*+}$}

Once the framework has been established using $D^{0}$ 's, it is a relatively straightforward extension to include $D^{*+}$ 's. After applying the $D^{*+}$ pion tag, the $K \pi$ signal to noise ratio is much larger, resulting in comparable statistical uncertainties despite the smaller number of events. Also, because the $\mu+D^{*}$ combination carries a slightly greater fraction of the parent $B$ momentum than just the $\mu+D^{0}$, the uncertainty of extrapolating back to the parent $B$ momentum is somewhat smaller. Finally, in many $B \rightarrow \mu D^{0} X$ decay the $D^{0}$ is the daughter of a $D^{*}$, so the yield is expected to be large.

To get a $D^{*}$ sample, as mentioned before, we start with events where the difference in mass of the $K^{-} \pi^{+} \pi^{+}$and $K^{-} \pi^{+}$systems is less than $153 \mathrm{MeV}$. Next, we apply the following requirements to the $K \pi$ combination:

- $q(K)=q(\mu)$ (i.e. the charge is consistent with $B$ decay)

- $m(\mu K \pi) \leq 5.3 \mathrm{GeV}(m(B))$

- $p_{T}(K) \geq 1.5 \mathrm{GeV}$ 
- $p_{T}(\pi)$ from $D^{0} \rightarrow K \pi \geq 1.5 \mathrm{GeV}$

- $p_{T}(\pi)$ from $D^{*} \rightarrow D \pi \geq 450 \mathrm{MeV}$

- $p_{T}(K)$ or $p_{T}(\pi)$ from $D^{0} \geq 3.0 \mathrm{GeV}$

Note that we have removed the $\cos \theta^{*}$ cut used in the $D^{0}$ analysis. Also note that we require the $D^{0}$ from the $D^{*+}$ decay to decay via $K^{-} \pi^{\text {r }}$, thus avoiding the difficulties of calculating the efficiencies and acceptances when the $D^{0}$ 's can decay via several channels, each with a different acceptance and efficiency. This sample is not statistically independent of the $D^{0}$ sample; $80 \%$ of the events in the $D^{*+}$ sample are also included in the $D^{0}$ sample. The $D^{*}$ candidate mass distribution with these cuts (for right sign muons) is shown in Figure 4 . Here the mass difference cut is relaxed and a $\pm 30 \mathrm{MeV}$ cut is imposed on the $D$ candidate mass.

The fitting procedure is similar to the $B \rightarrow \mu D^{0} X$; the only difference is that the wrong sign lepton background is replaced by a wrong sign lepton and pion background - that is, the background to $\left(K^{-} \pi^{+}\right) \pi^{+} \mu^{-}$is $\left(K^{-} \pi^{+}\right) \pi^{-} \mu^{+}$. In this way we compare two neutral objects instead of a $q=0$ object with a $q= \pm 2$ object. Figure 5 shows the $K \pi$ mass spectrum for the signal (top) and background (bottom) lepton-pion combinations. Finally, we reduce the number of $D^{*}$ tagged $D^{0}$ 's by $6 \pm 6 \%$ to account for the possibility of accidental tags: finding a $\pi$ that is not from a $D^{*}$ that happens to pass all the $D^{*}$ tagging requirements. This value comes from counting the number of $D^{*}$ tags in a different mass difference window, one that has the same number of pions as in the $D^{*}$ mass window. As before, we also divide the sample into three bins of $p_{T}$.

The number of reconstructed $D^{*+}$ 's and the $B$ cross section from this number are shown below.

\begin{tabular}{||l|r|r||}
\hline$p_{T}$ range & Events reconstructed & $d \sigma / d p_{T}|y|<1(\mathrm{nb} / \mathrm{GeV})$ \\
\hline All & $153 \pm 20$ & - \\
\hline $18-22 \mathrm{GeV}$ & $39 \pm 9$ & $10.8 \pm 2.6 \pm 1.9$ \\
\hline $22-26 \mathrm{GeV}$ & $43 \pm 8$ & $5.9 \pm 1.2 \pm 1.1$ \\
\hline $26-34 \mathrm{GeV}$ & $27 \pm 7$ & $1.2 \pm 0.3 \pm 0.2$ \\
\hline
\end{tabular}

The sources of systematic uncertainty are cnumerated in the following table. 


\begin{tabular}{||l|r||}
\hline Source of Uncertainty & Uncertainty \\
\hline Luminosity & $3.6 \%$ \\
\hline Acceptance & $6 \%$ \\
\hline Tracking efficiency & $7 \%$ \\
\hline Trigger efficiency & $3 \%$ \\
\hline$K, \pi$ decays in flight & $2 \%$ \\
\hline$B p_{T}$ resolution & $4 \%$ \\
\hline Fitting method and background shapc & $8 \%$ \\
\hline Branching ratio $(B \rightarrow \mu X)$ & $10 \%$ \\
\hline Branching ratio $\left(B \rightarrow \mu D^{* *}\right)$ & $2 \%$ \\
\hline Branching ratio $\left(D^{*+} \rightarrow \pi^{+} D\right)$ & $2 \%$ \\
\hline Branching ratio $(D \rightarrow K \pi)$ & $6 \%$ \\
\hline Total & $18 \%$ \\
\hline
\end{tabular}

\section{$4 \quad B_{s}$ Fraction}

The same technique of measuring the $B$ cross section by reconstructing non-strange $D$ mesons in association with muons could, in principle, be used to measure the $B_{s}$ cross section by reconstructing $D_{s}$ mesons produced in association with muons. Because the $B_{s}$ production rate is smaller than the non-strange $B$ production rate, and because the combined branching ratio $B R\left(D_{s} \rightarrow \phi \pi\right) \times B F\left(\phi \rightarrow K^{+} K^{-}\right)$is smaller than $B R\left(D^{\circ} \rightarrow K \pi\right)$ there are not nearly as many reconstructed $\mu+D_{s}$ events as $\mu+D^{0}$ events.

We therefore turn to a different method. We expand our sample to include electrons as well, and apply rather tight isolation cuts on the lepton. In the $\phi \pi$ spectrum, the $D_{s}$ and the (Cabibbo suppressed) $D^{+}$peaks are visible. By measuring the ratio of observed $D_{s}$ 's (coming predominantly from $B_{s}$ decays) to $D^{+}$'s (coming predominantly from $B^{-}$or $\bar{B}^{0}$ decays), we can extract the ratio of produced $B_{s}$ 's relative to $B^{0}$ or $B^{+ \text {'s. }}$.

The muon data used in this analysis begins with the data used the $\mu+D^{0}$ or $D^{*}$ analysis, with the trigger requirement removed and with the $p_{T}$ cut lowered to $6 \mathrm{GeV}$. Additionally we also include approximately $3 p b^{-1}$ of prescaled $6 \mathrm{GeV}$ muon triggers. The electrons used passed the following requirements:

- $E_{T}$ (electron) $\geq 6 \mathrm{GeV}$

$\bullet 0.75<E / p<1.5$, where $E / p$ is the ratio of the energy deposited by the electron candidate in the electromagnetic calorimeter to the momentum of the electron 
candidate, measured in the tracking chamber.

- $E$ (hadronic)/ $E$ (electromagnetic) The ratio energy in the hadronic calorimeter to the electromagnetic calorimeter be less the 0.1 If there is only one track pointing at the calorimeter tower (the electron) this cut is tightened to 0.04 .

- Track match to the calorimeter cluster: $\Delta x<1.5 \mathrm{~cm}, \Delta z<3.0 \mathrm{~cm}$

- The shower shape at shower maximum be consistent with an electromagnetic shower: $\chi^{2}$ (strips) $<10$ and $\chi^{2}$ (wires) $<15$

- Identified photon conversions are removed.

Finally, all leptons are required to be in the SVX fiducial region. This results in a sample of approximately 350,000 muon and 850,000 electron candidates.

From this sample, we reconstruct $D_{s} \rightarrow \phi \pi$ and $D^{+} \rightarrow \phi \pi$ in the following manner. Again we begin with the lepton as a seed, then tracks in a cone of $R(\equiv$ $\left.\sqrt{(\Delta \phi)^{2}+(\Delta \eta)^{2}}\right)<0.8$ around the lepton are selected for further analysis. Two tracks from oppositely charged particles are assigned the kaon mass, and if their invariant mass is within $\pm 8 \mathrm{MeV}$ of the $\phi$ mass of $1019 \mathrm{MeV}$, the combination is classified as a $\phi$ candidate. Next, the $\phi$ is combined with another track that is assigned the pion mass and the three track combination is fit, with the constraint that all three tracks intersect at a common point with a probability $P\left(\chi^{2}\right) \geq 1 \%$. We accept the combination if the following requirements are met:

- $p_{T}(K) \geq 1 \mathrm{GeV}$

- $p_{T}(\pi) \geq 800 \mathrm{MeV}$

- $p_{T}(\phi) \geq 2 \mathrm{GeV}$

- The lepton is isolated, where an isolated lepton is defined as one where the transverse energy in a cone of $R \leq 0.4$ around the lepton does not exceed the $\phi \pi$ transverse momentum by more than $20 \%$.

- The displacement of the $\phi \pi$ vertex projected along the momentum direction is positive. That is, the vertex from the charm decay is not behind the primary vertex.

Figure 6(top) shows the $\phi \pi$ mass distribution where the associated lepton had a charge consistent with $B$ decay, (e.g. $B_{s} \rightarrow D_{s}^{-} e^{+} \nu$ ) and (bottom) the distribution where the lepton had the wrong charge. Peaks from both $D_{s} \rightarrow \phi \pi$ and $D^{+} \rightarrow \phi \pi$ are visible. We fit the ratio of the number of $D_{s}$ mesons observed to the number of $D^{+}$'s and find it to be $3.4_{-1.0}^{+2.4}$ for the electron sample, $4.1_{-1.3}^{+3.0}$ for the muon sample, and $3.5_{-1.1}^{+2.3}$ for the combined muons and electron sample. 
The relationship between the cross section and the number of reconstructed $D^{+}$ and $D_{s}$ mesons (the acceptance and reconstruction efficiency of the two states is equal to within $10 \%$ ) is given by:

$$
\frac{N\left(D_{s} \rightarrow \phi \pi\right)}{N\left(D^{+} \rightarrow \phi \pi\right)}=\frac{\sigma\left(B_{s}\right)}{\sigma\left(B_{u, d}\right)} \frac{B R\left(B_{s} \rightarrow D_{s} l X\right)}{B R\left(B_{u, d} \rightarrow D^{+} l X\right)} \frac{B R\left(D_{s} \rightarrow \phi \pi\right)}{B R\left(D^{+} \rightarrow \phi \pi\right)}
$$

or

$$
\frac{\sigma\left(B_{s}\right)}{\sigma\left(B_{u, d}\right)}=\frac{\Gamma\left(B_{u, d} \rightarrow D^{+} l X\right)}{\Gamma\left(B_{s} \rightarrow D_{s} l X\right)} \frac{\tau\left(B_{s}\right)}{\tau\left(B_{u, d}\right)} \frac{B R\left(D^{+} \rightarrow \phi \pi\right)}{B R\left(D_{s} \rightarrow \phi \pi\right)} \frac{N\left(D_{s} \rightarrow \phi \pi\right)}{N\left(D^{+} \rightarrow \phi \pi\right)}
$$

where the branching fractions have been replaced by partial widths and lifetimes.

We assume that the semileptonic partial widths for all $B_{q}$ mesons are the same, and that the lifetimes are equal to within $12 \%$. Additionally, we assume that the $D^{* *}$ fraction is the same $(20 \pm 10 \%)$ in all decays and that all $D_{s}^{* *}$ s decay to $D K$. From these assumptions and the branching fractions $D^{+} \rightarrow \phi \pi^{+}$of $0.57 \pm 0.11 \%[3]$ and $D_{s} \rightarrow \phi \pi^{+}$of $5.1 \pm 0.9 \%$ [4] we can calculate the relative fraction of $B_{s}$ mesons:

$$
\frac{\sigma\left(B_{s}\right)}{\sigma\left(B_{u}\right)+\sigma\left(B_{d}\right)}=.26_{-.08}^{+.17} \pm .08
$$

The systematic uncertainties are dominated by branching ratios and are enumerated in the following table.

\begin{tabular}{||l|r||}
\hline Source of Uncertainty & Uncertainty \\
\hline$B \rightarrow D, D^{*} l X$ branching ratio & $10 \%$ \\
\hline$B \rightarrow D^{* *} l X$ branching ratio & $10 \%$ \\
\hline$D_{s} \rightarrow \phi \pi$ branching ratio & $18 \%$ \\
\hline$D^{+} \rightarrow \phi \pi$ branching ratio & $13 \%$ \\
\hline Relative acceptance and efficiency for $D_{s}$ and $D^{+}$ & $10 \%$ \\
\hline$\tau\left(B_{s}\right) / \tau\left(B_{u, d}\right)$ & $12 \%$ \\
\hline Total & $31 \%$ \\
\hline
\end{tabular}

\section{Summary}

The $B$ meson cross section has been measured by reconstructing $D^{0}$ and $D^{*+}$ mesons in association with muons. This technique allows the backgrounds to be measured, as 
opposed to calculated. The results are compared to the lower $p_{T}$ CDF measurements from $B^{+} \rightarrow J / \psi K^{+}$and $B^{0} \rightarrow J \psi K^{* 0}$ as well as the Next-to-Leading Order QCD prediction using the MRSD0 parton distributions in Figure 7.

The CDF $B_{s}$ to $B_{u, d}$ fraction is consistent with the popular assumption that $15 \%$ of $b$ 's fragment into $B_{s}$ and $75 \%$ into $B_{u}$ or $B_{d}$. It is slightly larger than the LEP average [5] of $.112 \pm .024 \perp .020$ when one includes the new CLEO $D_{s}$ branching fraction.

We thank the Fermilab staff and the technical staffs of the participating institutions for their vital contributions. This work was supported by the U.S. Department of Energy and National Science Foundation; the Italian Istituto Nazionale di Fisica Nucleare; the Ministry of Education, Science and Culture of Japan; the Natural Sciences and Engineering Research Council of Canada; the National Science Council of the Republic of China; the A. P. Sloan Foundation; and the Alexander von Humboldt-Stiftung.

\section{References}

[1] F. Abe et al., Nucl. Instr. and Meth. A271 (1988) pp.387. Also F. Abe et al. FERMILAB-PUB-94/097-E

[2] To be published in the proceedings of the XXIXth Rencontres de Moriond, QCD and High Energy Hadronic Interactions (1994) Also FERMILAB-CONF-94/113$\mathrm{E}$

[3] Particle Data Group

[4] F. Butler et al., Phys. Lett. B324 (1994) p.255. Different measurements of this branching fraction show substantial variations.

[5] W. Venus, rapporteur talk at the XVI International Symposium on LeptonPhoton Interactions. (1993) Also Phys. Lett. B294 p.145, Phys. Lett. B289 p.199, Phys. Lett. B295 p.357. 


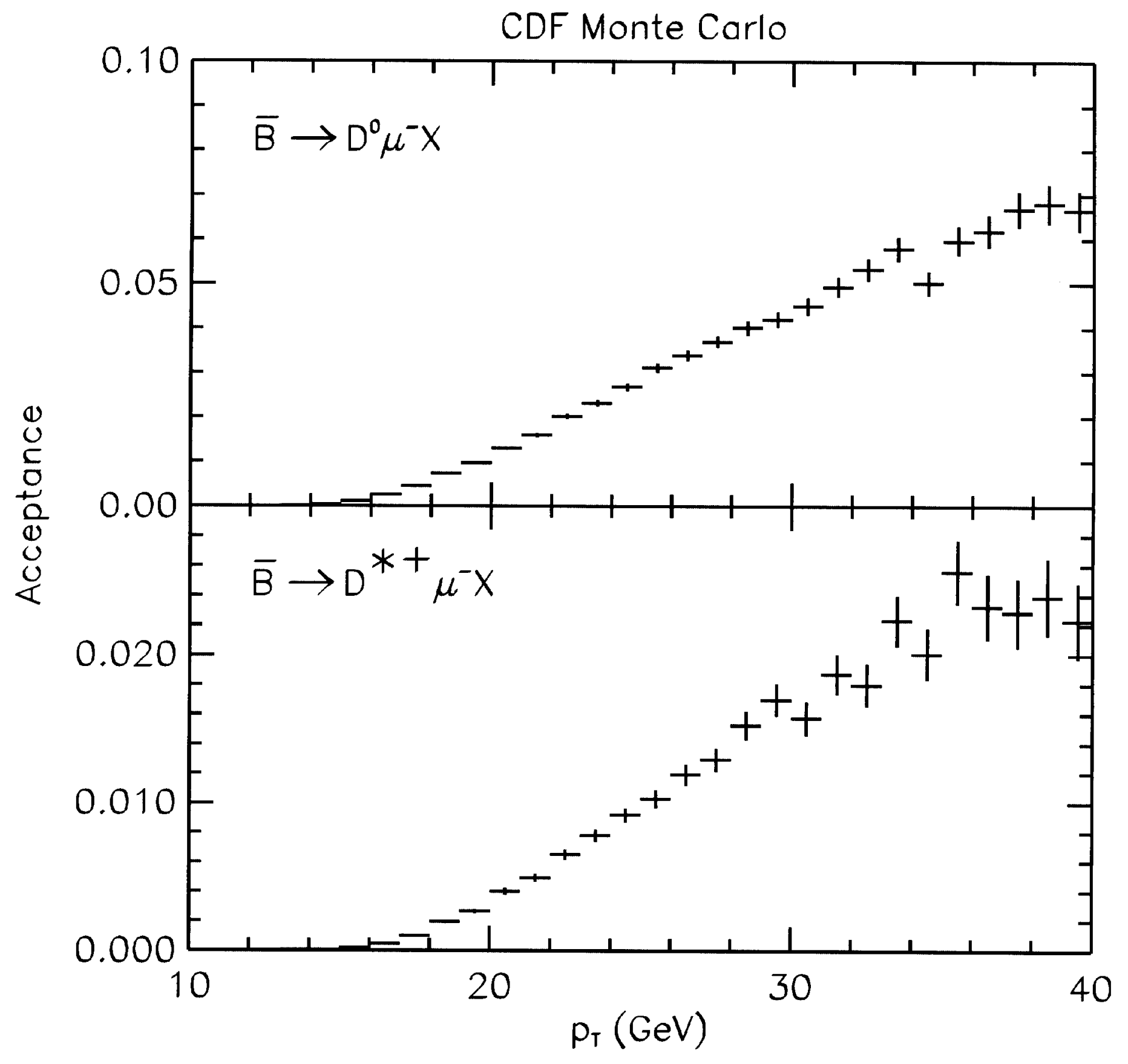

Figure 1: Generated/Corrected $B$ meson $p_{T}$ vs. Generated $B p_{T}$. 
CDF Monte Carlo

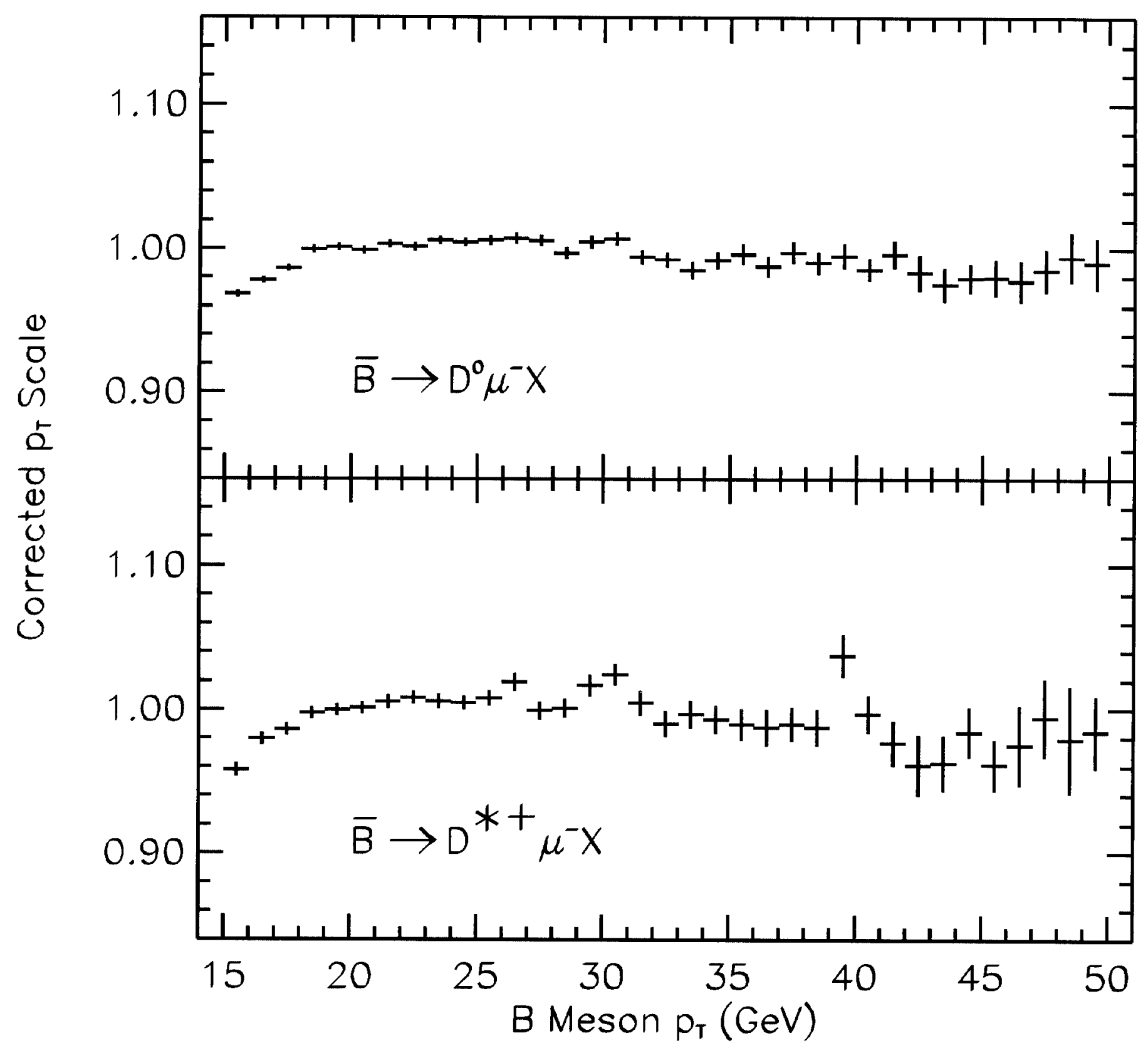

Figure 2: Acceptance and Reconstruction Efficiency vs. $p_{T}$. 


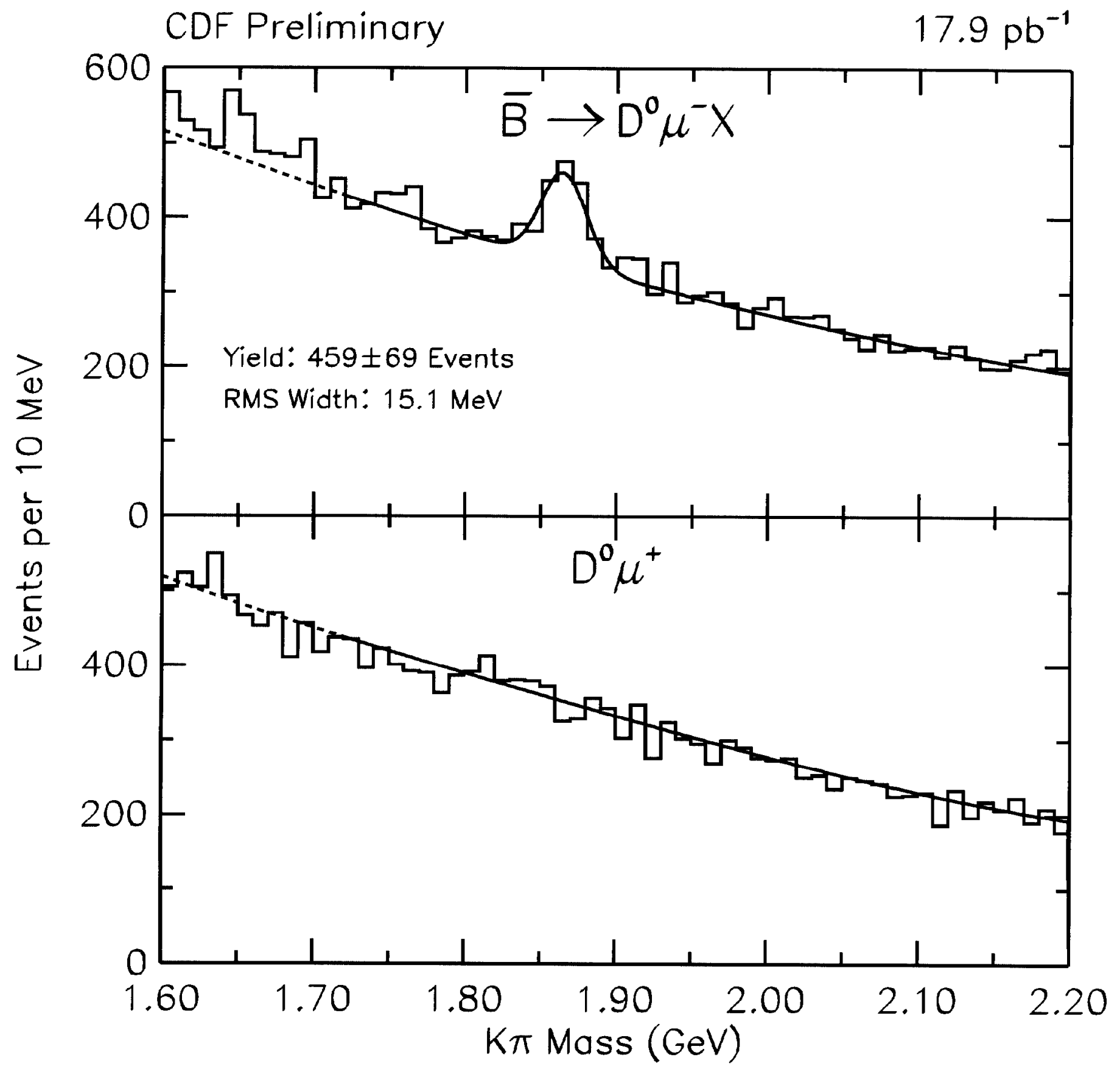

Figure 3: $D^{0}$ from $B \rightarrow \mu D^{0} X$. 


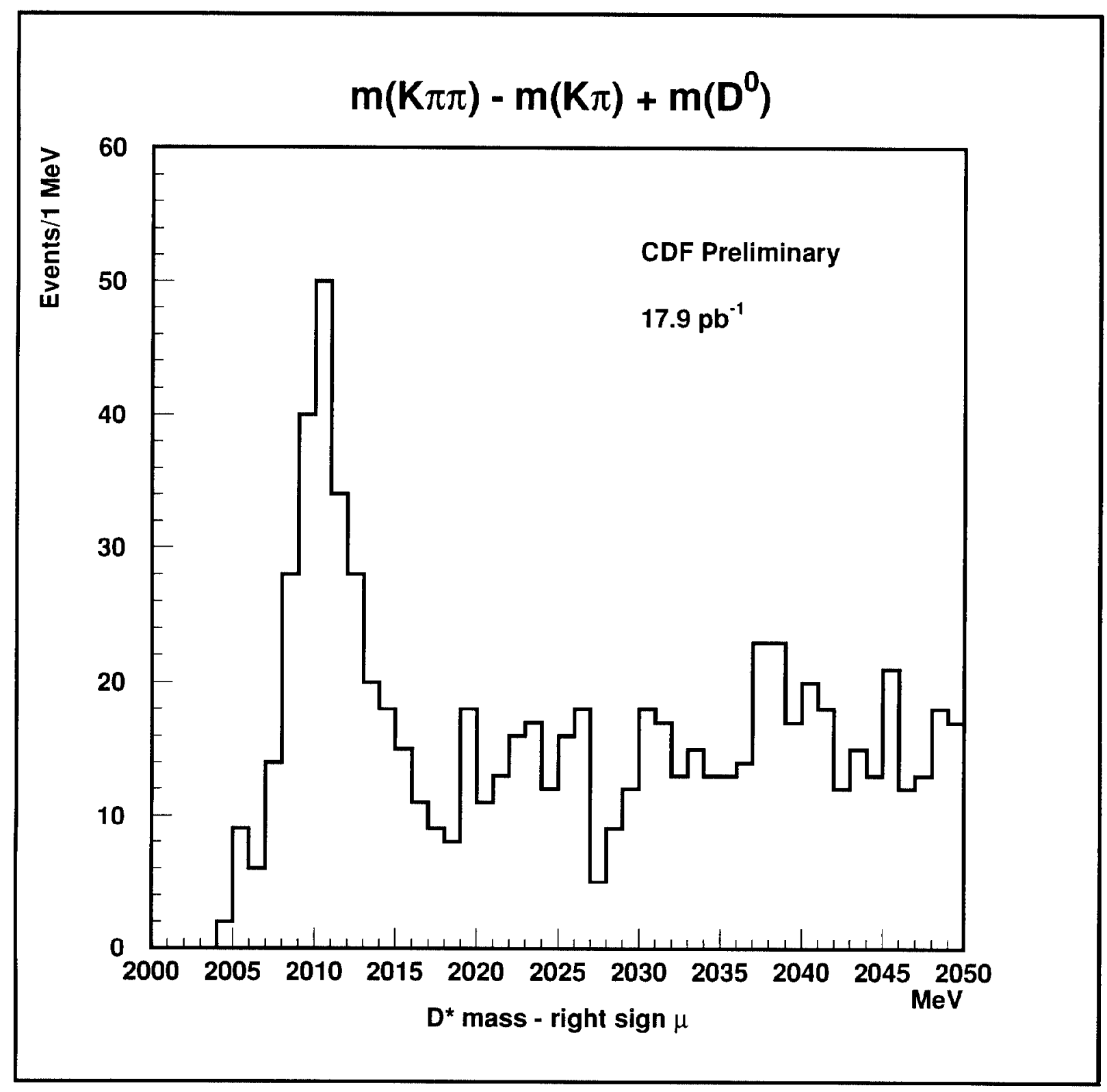

Figure 4: $D^{*}-D^{0}$ mass difference. 


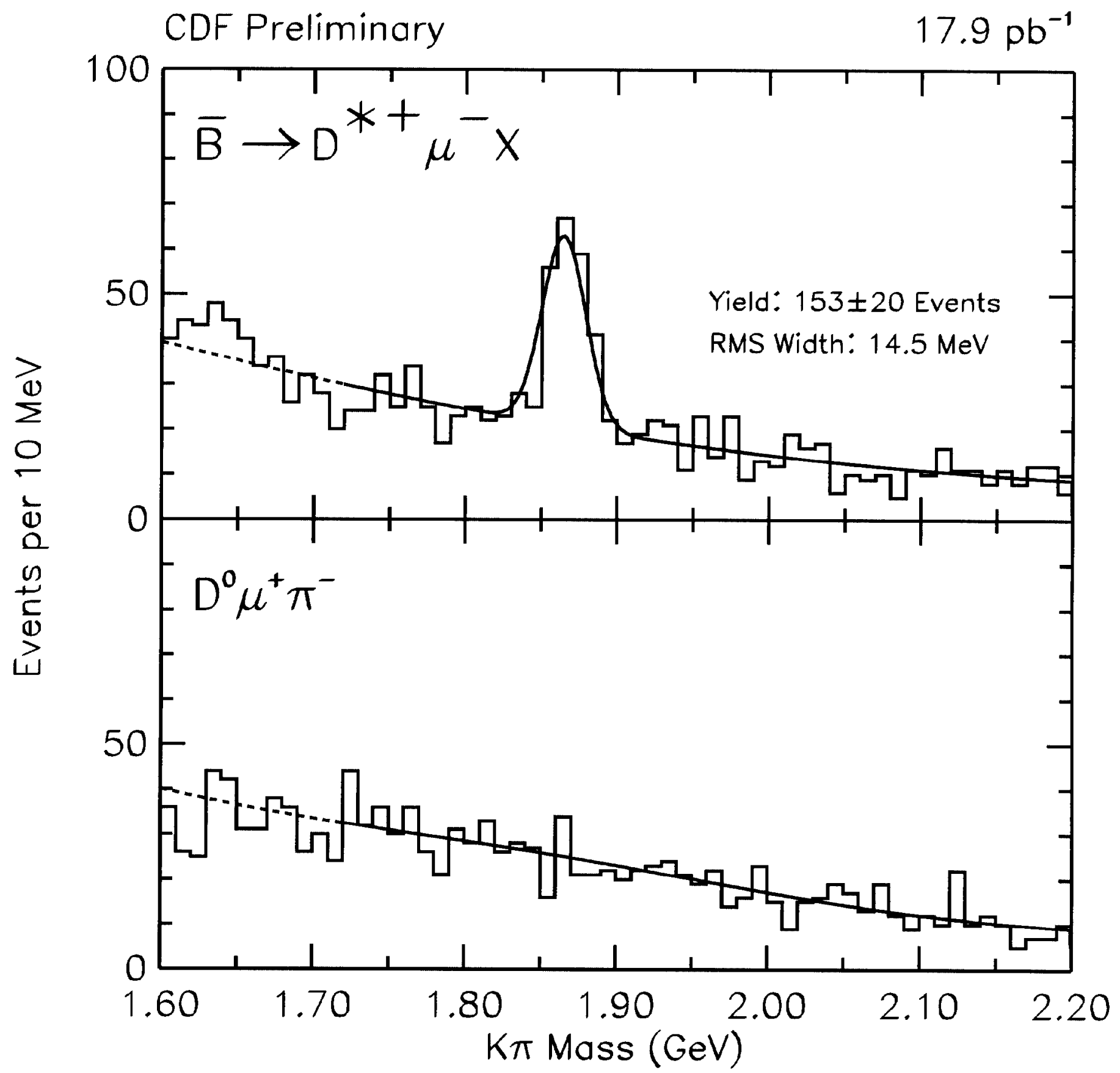

Figure 5: $D^{*+}$ from $B \rightarrow \mu D^{*+} X$. 


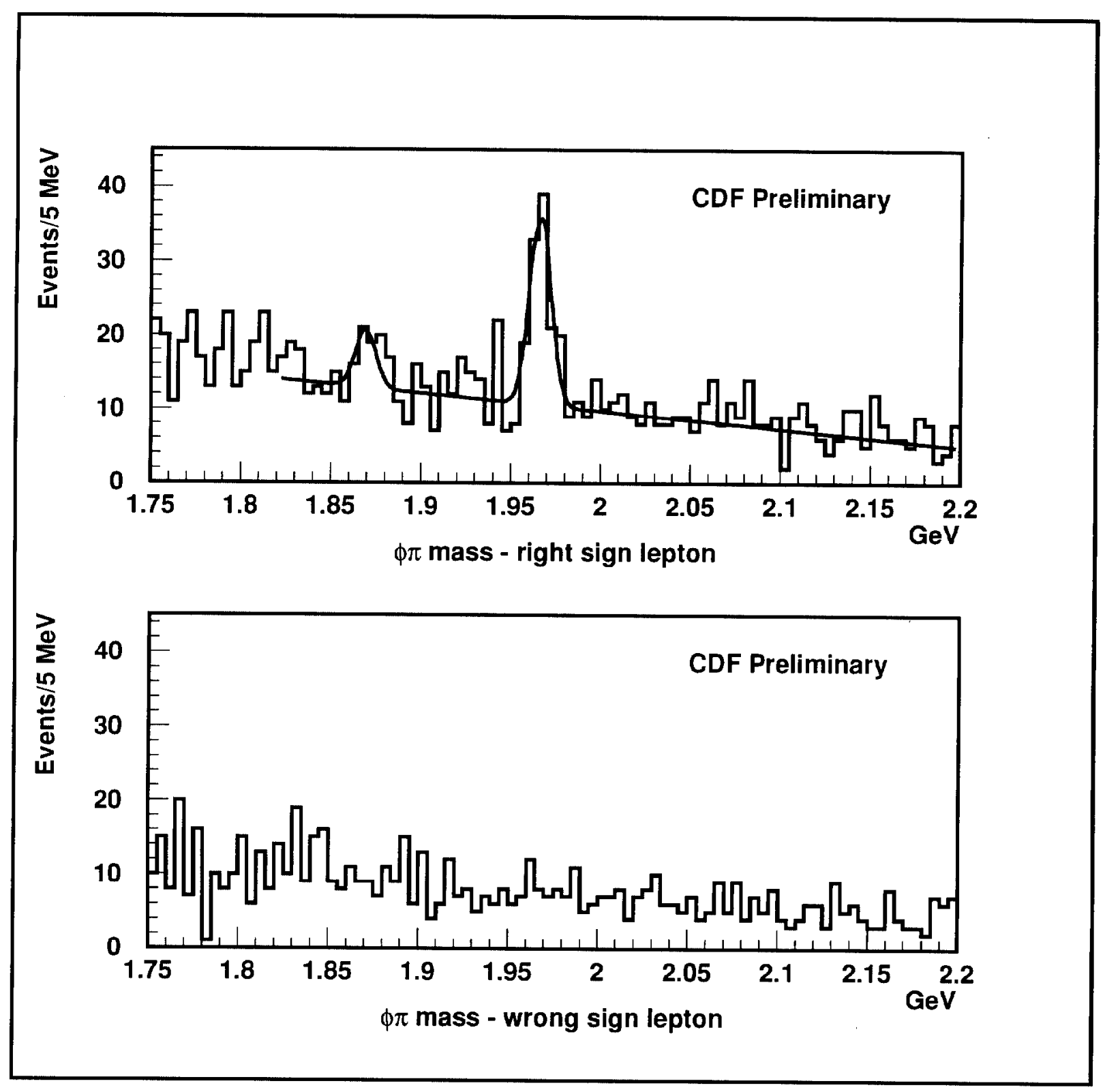

Figure 6: $\phi \pi$ mass spectrum in combined $e$ and $\mu$ sample. 


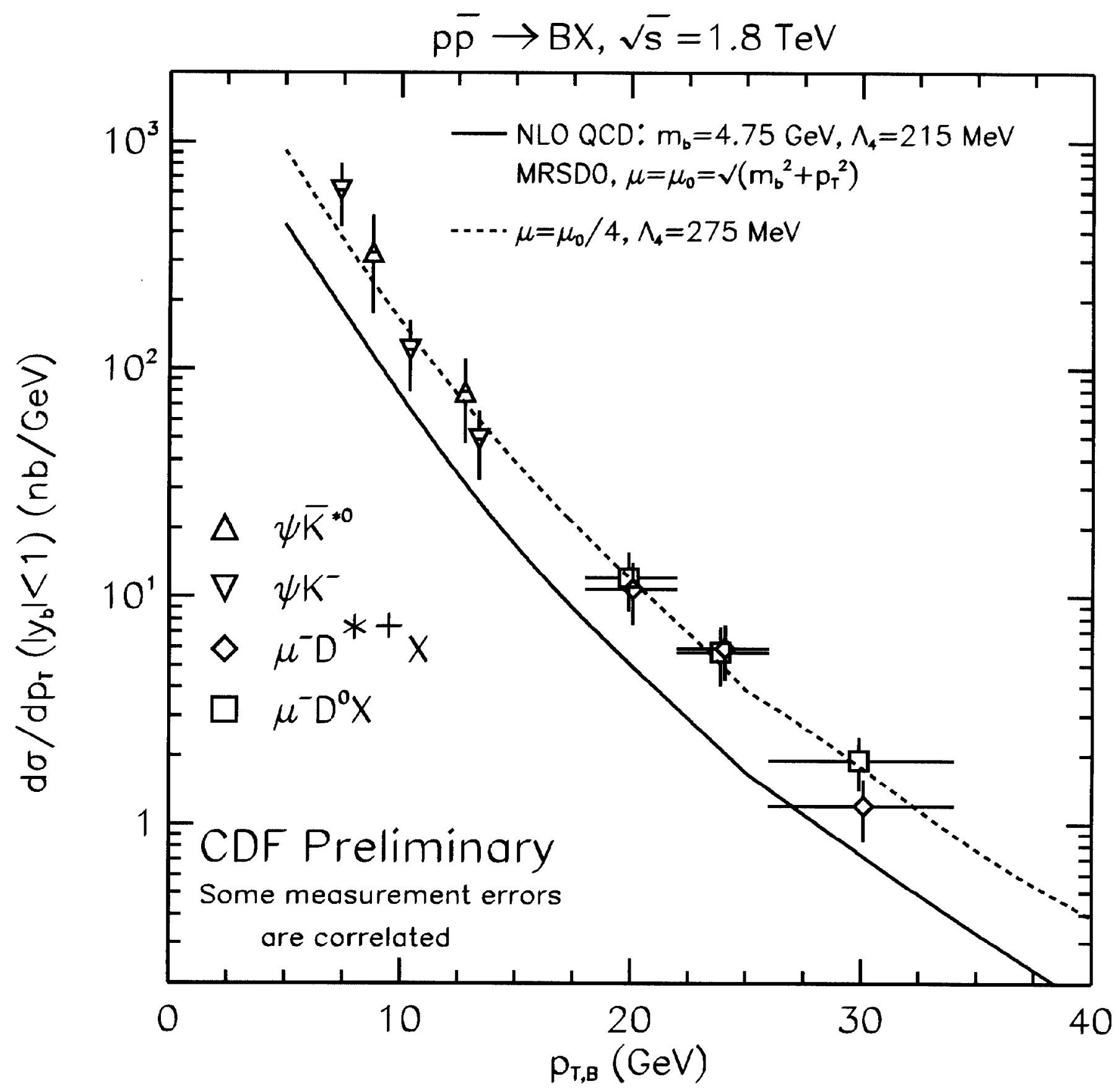

Figure 7: Selected CDF $B$ cross section measurements. 